\title{
THE FELINE JOSEPHUS PROBLEM
}

\author{
FRANK RUSKEY AND AARON WILLIAMS
}

\begin{abstract}
In the classic Josephus problem, elements $1,2, \ldots, n$ are placed in order around a circle and a skip value $k$ is chosen. The problem proceeds in $n$ rounds, where each round consists of traveling around the circle from the current position, and selecting the $k$ th remaining element to be eliminated from the circle. After $n$ rounds, every element is eliminated. Special attention is given to the last surviving element, denote it by $j$. We generalize this popular problem by introducing a uniform number of lives $\ell$, so that elements are not eliminated until they have been selected for the $\ell$ th time. We prove two main results: 1) When $n$ and $k$ are fixed, then $j$ is constant for all values of $\ell$ larger than the $n$th Fibonacci number. In other words, the last surviving element stabilizes with respect to increasing the number of lives. 2) When $n$ and $j$ are fixed, then there exists a value of $k$ that allows $j$ to be the last survivor simultaneously for all values of $\ell$. In other words, certain skip values ensure that a given position is the last survivor, regardless of the number of lives. For the first result we give an algorithm for determining $j$ (and the entire sequence of selections) that uses $O\left(n^{2}\right)$ arithmetic operations.
\end{abstract}

\section{"un gatto ha sette vite"}

\section{INTRODUCTION}

1.1. A Fanciful Scenario. In this subsection we describe some of the history that lead to the classic Josephus problem, and then invent a scenario that might have led to our version of the Josephus problem.

During the first Jewish-Roman war, the military leader Josephus (AD 37 - c. 100) and 40 of his countrymen hid from the Romans in the fallen city of Yodfat. With no hope for escape, the Jewish survivors agree to commit mass suicide rather than be captured and enslaved by the enemy. Josephus does not agree with the others, and instead convinces them to take part in a lethal game of chance:

"He whom the lot falls to first, let him be killed by him that hath the second lot, and thus fortune shall make its progress through us all." (Book 3, Chapter 8, Section 7 in The Jewish War [14].)

Whether by chance or "by the providence of God" Josephus survives this ordeal, and eventually becomes the Roman citizen and Jewish historian known as Titus Flavius Josephus.

By 1539, Girolamo Cardano described the situation as a mathematical puzzle [5]. Instead of drawing lots, the 41 men form a circle and then every 3rd person around the circle is successively selected for elimination. Using this interpretation, Josephus is recast as an erudite scholar who quickly determined that he should stand in the 31st position to avoid elimination.

Key words and phrases. Josephus problem, Fibonacci number, Chinese remainder theorem, Bertrand's postulate, number theory, algorithm.

Research supported in part by an NSERC discovery grant. 
In general, the classic Josephus problem has two parameters: $n$ and $k$. A circle of $n$ people is formed, and successively every $k$-th person is selected for elimination. As people are killed off, the circle shrinks, and the goal is to determine last surviving position $j$. In particular, Josephus solved the first instance of the problem by determining that $j=31$ when $n=41$ and $k=3$. In all versions of the problem, including ours, Josephus knows where the circle begins and has the right to stand in whatever position he wishes.

Now imagine that Josephus's countrymen agree with his orderly method of elimination, but suspect that Josephus has already determined where to stand. For this reason, they introduce a third parameter $\ell$ (for lives). Again the men form a circle, but this time they do not die until they have been selected (also called "hit") for the $\ell$-th time. Inspired by the Italian saying that "un gatto ha sette vite" (cats have seven lives) ${ }^{1}$, we call this generalization the Feline Josephus problem. In the original problem $\ell=1$.

To further complicate matters, Josephus's countrymen hide the value of $\ell$ from their military leader. Undeterred, Josephus agrees to this change of plans, but only under one of the following two conditions: (a) he gets to specify a lower bound on $\ell$, or (b) he gives up his right to specify where he stands, in exchange for choosing the value of $k$. Amazingly enough, Josephus continues to survive, and the main purpose of this paper is to tell the reader how he manages to do this.

In part, our motivation for studying (b) is the second bonus problem in Concrete Mathematics [10] which asks the following:

Suppose that Josephus finds himself in a given position $j$, but he has a chance to name the elimination parameter $k$ such that every $k$ th person is executed. Can he always save himself?

As we will show, the answer is yes, not only for $\ell=1$, but for any $\ell \geq 1$.

1.2. Notation. The parameters to the feline Josephus problem are $n$ (the number of people), $k$ (the skip factor), and $\ell$ (the number of "lives"). It is important to note that we make no assumptions about the parameters, other than that are positive integers. In particular, $k$ or $\ell$, or both, could be larger than $n$.

When the parameters are fixed, as they usually are, we introduce the following three notations. Let hit $(i)$ be the $i$ th person that is selected (or "hit"). The sequence of all successive hits is called the hit sequence; it is

$$
\operatorname{hit}(1), \operatorname{hit}(2), \ldots, \operatorname{hit}(\ell \cdot n) \text {. }
$$

Let $\operatorname{kill}(i)$ be the $i$ th person that is eliminated (or "killed"). Note that $j=\operatorname{kill}(n)=$ $\operatorname{hit}(\ell \cdot n)$ denotes the last surviving element. The kill sequence is

$$
\operatorname{kill}(1), \operatorname{kill}(2), \ldots, \operatorname{kill}(n) .
$$

Let $\operatorname{round}(i)$ be the sequence of selections that take place after (but not including) the $(i-1)$ person is eliminated, up to (and including) the selection that eliminates the $i$ th person. Thus the hit sequence may be written as

$$
\operatorname{round}(1), \operatorname{round}(2), \ldots, \operatorname{round}(n) .
$$

Finally, let $\ell_{r}(i)$ represent the remaining lives for person $i$ at the end of round $r$. In particular, $\ell_{0}(i)=\ell$ for all $i$, and the last surviving element is the unique value of $i$ such that $\ell_{n-1}(i)>0$.

\footnotetext{
${ }^{1}$ In some cultures the saying is "cats have nine lives."
} 

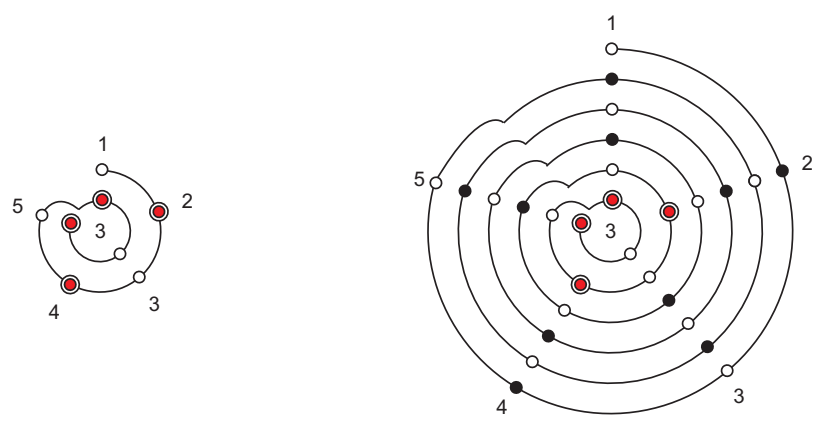

Figure 1. The Josephus problem with $n=5, k=2, \ell=1$ (left) and $\ell=3$ (right).

Example 1: Let $n=5, k=2$, and $\ell=1$. Then the hit sequence is

$$
2,4,1,5,3 \text {, }
$$

which is the same as the kill sequence. If we now set $\ell=3$, then we obtain the hit sequence

$$
\underbrace{2,4,1,3,5,2,4,1,3,5,2}_{\text {round }(1)}, 4,1,5,3,
$$

and the same kill sequence as before. The first round is indicated by the underbrace above; the other four rounds are singletons. This example is illustrated in Figure 1; it starts at the outer 1 and works inwards. In the figure white filled dots are persons passed over and not hit, filled dots are persons hit, and a filled red dot is a person hit for the last time. The last survivor, person 3 , is recorded in the center.

The reason that we did not continue with the circular arcs is that they would have spiralled around several times before hitting 3. In general it is desirable to avoid spiralling around the circle without hitting anybody. In round $r$ this will happen if $k>n-r+1$. Obviously the useless spirals can be eliminated by using $k \bmod n-r+1$ in round $r$ instead of $k$; to save computation, this will be done later on in an algorithm.

One consequence of the observation in the above paragraph is that we may always reduce $k$ to $k \bmod \operatorname{lcm}\{1,2, \ldots, n\}$ without changing the hit sequence. In the OEIS $\operatorname{lcm}\{1,2, \ldots, n\}$ is sequence A003418 [19]. The prime number theorem implies that $\operatorname{lcm}\{1,2, \ldots, n\} \sim e^{(1+o(1))}$, so it grows much slower than $n$ !.

Example 1 is trivial in a sense, because $n$ and $k$ are relatively prime, (which we denote $n \perp k$, following [10]). For larger values of $\ell$ in the example above, the hit sequence will be $(2,4,1,3,5)^{\ell-1}, 2,4,1,5,3$. More generally, whenever $n \perp k$ the hit sequence will have the form $\pi^{\ell-1} \tau$ where $\pi$ and $\tau$ are permutations of $\{1,2, \ldots, n\}$ and $\tau$ is the kill sequence for $\ell=1$. Thus, the new problem is only interesting when $n$ and $k$ are not relatively prime. In particular, $41 \perp 3$ so the original instance of the Josephus problem would have been no more interesting with cats replacing humans. Next we will consider an example in which $n \not \perp k$. 

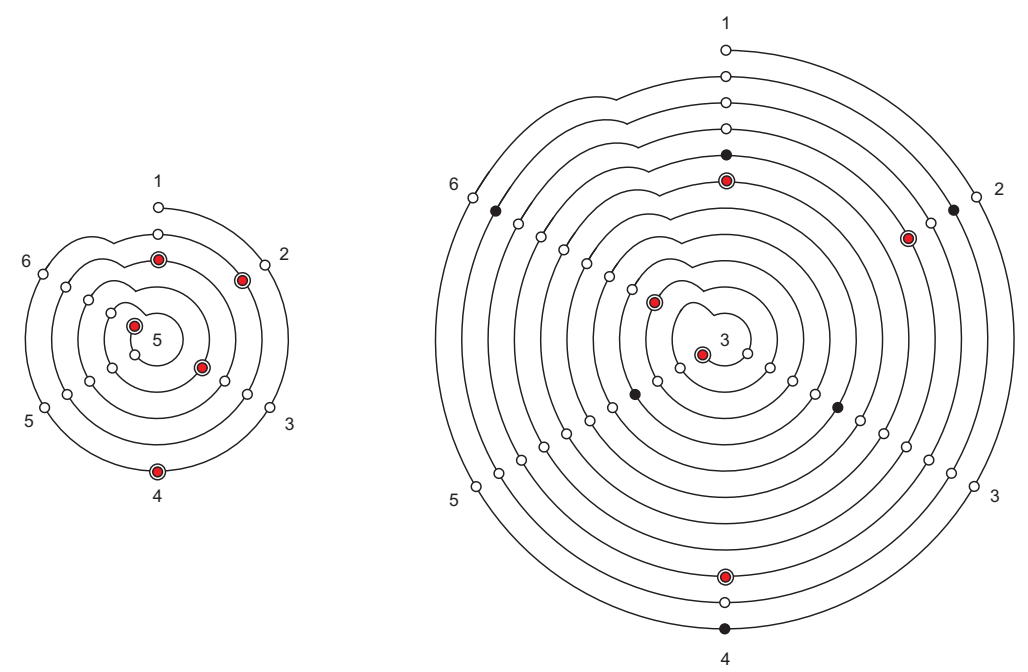

Figure 2. The Josephus problem with $n=6, k=4, \ell=1$ (left) and $\ell=2$ (right).

Example 2: In this example $n=6$ and $k=4$. See Figure 2. If $\ell=1$, then the hit sequence and the kill sequence are both $4,2,1,3,6,5$. If $\ell=2$, then the hit sequence is $4,2,6,4,2,1,1,3,5,6,5,3$, and the kill sequence is $4,2,1,6,5,3$ which is different from the kill sequence when $\ell=1$.

1.3. History. There are several related research problems for the original Josephus problem: characterizing and computing the last survivor $\operatorname{kill}(n)[15,16,9]$, or the $i$ th-last surviving element $\mathrm{kill}(n-i+1)[13,21,11,20]$, studying the combinatorial properties of $\operatorname{kill}(1) \operatorname{kill}(2) \cdots \operatorname{kill}(n)$ as a permutation of $\{1,2, \ldots, n\}[17,7,3,6]$, when considering $\operatorname{kill}(n-r+1), \operatorname{kill}(n-r+2), \ldots, \operatorname{kill}(n)$ as an $r$-element subset of $\{1,2, \ldots, n\}[10]$ (especially when $r=n / 2$ [8]). The problem plays an interesting role in the history of combinatorics [2], and is used for mathematical recreation [1] and education [12, 10, 18]. Variations have also been examined $[4,20]$, but the feline version of the problem appears to be new.

\section{Josephus meets Leonardo of Pisa}

When $n$ and $k$ have a common factor, then the value of the last survivor is contingent upon the value of $\ell$. As shown in Example 2, when $n=6$ and $k=4$, the last surviving element is $\operatorname{kill}(n)=5$ when $\ell=1$, and changes to $\operatorname{kill}(n)=3$ when $\ell=2$.

Example 3: For a more involved example, the values of $\operatorname{kill}(n)$ appears below for $n=12$, $k=14642$, and $\ell=1,2, \ldots, 10$ respectively:

$$
\operatorname{kill}(n)=9,1,11,11,11,5,5,5,1,1 .
$$

Given this example, it is natural to fix $n$ and $k$, and to consider the value of $\operatorname{kill}(n)$ as $\ell \rightarrow \infty$. A priori, the ultimate behavior of $\operatorname{kill}(n)$ is not clear: it could continue to fluctuate chaotically, or could settle into a repeating pattern. The main result of this section is that there is a single limiting value for $\operatorname{kill}(n)$. Furthermore, this limiting value holds whenever $\ell$ exceeds the $(n+2)$ nd Fibonacci number.

To prove the result, we show that the value of $\ell_{r}(i)$ (the remaining lives for element $i$ at the end of round $r$ ) is contained in either an "increasing" set or a "decreasing" set. By ensuring that these two sets do not overlap, it is possible to give an explicit formula for each round $(r)$ 
(the hits between the $(r-1)$ st and $r$ th elimination). One consequence of this formula is the value of the last surviving element. Define the increasing set and decreasing set respectively as

$$
\mathcal{I}(r)=\left\{0,1,2, \ldots, F_{r}\right\} \text { and } \mathcal{D}(r)=\left\{\ell, \ell-1, \ldots, \ell-F_{r+1}+1\right\},
$$

where $F_{i}$ denotes the $i$ th Fibonacci number. As usual the Fibonacci numbers are defined as, $F_{0}=0, F_{1}=1$, and $F_{i}=F_{i-2}+F_{i-1}$ for all $i \geq 2$. Note that

$$
\mathcal{I}(0) \subseteq \mathcal{I}(1) \subseteq \cdots \subseteq \mathcal{I}(n) \quad \text { and } \quad \mathcal{D}(0) \subseteq \mathcal{D}(1) \subseteq \cdots \subseteq \mathcal{D}(n)
$$

Lemma 1. Let $n$ and $k$ be fixed positive integers. Then for any $i$ satisfying $1 \leq i \leq n$ and any $r$ satisfying $0 \leq r \leq n$,

$$
\ell_{r}(i) \in \mathcal{I}(r) \cup \mathcal{D}(r) .
$$

Proof. Our proof is by induction on $r$. The result is true when $r=0$ since $\ell_{0}(i)=\ell$ and $\mathcal{D}(0)=\{\ell\}$. Now assume that (1) holds for all rounds previous to round $r$. We will use this assumption to prove that (1) holds for round $r$.

When $\mathcal{I}(r)$ and $\mathcal{D}(r)$ have a value in common then (1) certainly holds since the union of these two sets includes $\{0,1, \ldots, \ell\}$. Therefore, we may assume that the maximum value of $\mathcal{I}(r)$ is strictly less than the minimum value of $\mathcal{D}(r)$. It is also safe to ignore the case when $i$ has been eliminated, since then $\ell_{r}(i)=0$ and (1) clearly holds. Similarly, if $i$ does not appear in $\operatorname{round}(r)$ then (1) holds by induction.

In the remaining case, let $y=\operatorname{kill}(r)$. Notice that $\ell_{r-1}(y) \leq \ell_{r-1}(i)$. Furthermore, $i$ must appear in round $(r)$ either the same number of times as $y$, or one time fewer than $y$. That is,

Inductively,

$$
\ell_{r}(i) \in\left\{\ell_{r-1}(i)-\ell_{r-1}(y), \ell_{r-1}(i)-\ell_{r-1}(y)+1\right\} .
$$

$$
\left\{\ell_{r-1}(i), \ell_{r-1}(y)\right\} \subseteq \mathcal{I}(r-1) \cup \mathcal{D}(r-1)
$$

and so there are four cases depending on which sets contain these two values. However, it is not possible for $\ell_{r-1}(i) \in \mathcal{I}(r-1)$ and $\ell_{r-1}(y) \in \mathcal{D}(r-1)$ since $\ell_{r}(y) \leq \ell_{r}(i)$ and our assumption that the maximum value of $\mathcal{I}(r)$ is strictly less than the minimum value of $\mathcal{D}(r)$. Furthermore, if $\ell_{r-1}(i) \in \mathcal{I}(r-1)$ and $\ell_{r-1}(y) \in \mathcal{I}(r-1)$ then obviously $\ell_{r}(i) \in \mathcal{I}(r-1)$ and so (1) follows from $\mathcal{I}(r-1) \subseteq \mathcal{I}(r)$. This leaves two cases to consider.

If $\ell_{r-1}(i) \in \mathcal{D}(r-1)$ and $\ell_{r-1}(y) \in \mathcal{I}(r-1)$ then

$$
\begin{aligned}
\ell_{r}(i) & \geq \min (\mathcal{D}(r-1))-\max (\mathcal{I}(r-1)) \\
& =\ell-F_{r}+1-F_{r-1} \\
& =\ell-F_{r+1}+1 \\
& =\min (\mathcal{D}(r)) .
\end{aligned}
$$

On the other hand, if $\ell_{r-1}(i) \in \mathcal{D}(r-1)$ and $\ell_{r-1}(y) \in \mathcal{D}(r-1)$ then

$$
\begin{aligned}
\ell_{r}(i) & \leq \max (\mathcal{D}(r-1))-\min (\mathcal{D}(r-1))+1 \\
& =\ell-\left(\ell-F_{r}+1\right)+1 \\
& =F_{r} \\
& =\max (\mathcal{I}(r)) .
\end{aligned}
$$

Therefore, $\ell_{r}(i) \in \mathcal{I}(r) \cup \mathcal{D}(r)$ as claimed, and so (1) is true by induction. 
Lemma 1 proved that the remaining number of lives at the end of each round are always contained in the increasing or the decreasing set. The next lemma says that these two sets are disjoint, so long as $\ell$ is chosen to be large enough.

Lemma 2. If $\ell \geq F_{n+2}$, then the maximum value in $\mathcal{I}(r)$ is less than the minimum value in $\mathcal{D}(r)$ for all $0 \leq r \leq n$.

Proof.

$$
\begin{aligned}
\max (\mathcal{I}(r)) & =F_{r} \\
& =F_{r+2}-F_{r+1} \\
& <F_{r+2}-F_{r+1}+1 \\
& \leq \ell-F_{r+1}+1 \\
& =\min (\mathcal{D}(r)) .
\end{aligned}
$$

2.1. Algorithmic Implications. Assuming the $\ell$ is large enough, we will now show that it is possible to represent the hit sequence by a data structure that uses $O\left(n^{2}\right)$ space and that can be constructed in $O\left(n^{2}\right)$ time. For example, let us consider the scenario discussed at the beginning of this section. When $n=12, k=14642$, and $\ell \geq 9$, we will show below that the hit sequence for the feline Josephus problem is the following sequence of length $n \ell=12 \cdot \ell$.

$$
\begin{aligned}
(2,4,6,8,10,12)^{\ell-1}, 2,3,4,6,3,1,12,(3,7,9,11)^{\ell-3}, 3 \\
10,(1,7,9)^{2}, 1,7,9,1,8,(1,11,5)^{2}, 1,11,5^{\ell-2}, 1^{\ell-8}
\end{aligned}
$$

Notice that this sequence immediately implies that the last surviving element is 1 whenever $\ell \geq 9$. The remainder of this section describes how sequences of this form can be constructed algorithmically. The strings are guaranteed to represent the hit sequence for all $\ell \geq F_{n+2}$, although the above example illustrates that the string often represents the hit sequence for smaller values of $\ell$ as well.

Recall that the hit sequence is obtained by concatenating the sequences $\operatorname{round}(r)$ for $r=1,2, \ldots, n$. We will show that the sequence for round $(r)$ can be expressed as $\pi^{h} \tau$ where $\pi$ is a sequence of unique elements from $\{1,2, \ldots, n\}$, as is $\tau$. Either $\pi$ or $\tau$ may be empty.

Let us first consider the problem of constructing a table of the values of $\ell_{r}(i)$ for $i=$ $1,2, \ldots, n$ and $r=0,1, \ldots, n$. This process is best illustrated by an example, which is the same as the one that started this subsection. The following table shows the values of $n-r+1 \bmod 14642$.

\begin{tabular}{c|cccccccccccc}
$\bmod$ & 12 & 11 & 10 & 9 & 8 & 7 & 6 & 5 & 4 & 3 & 2 & 1 \\
\hline 14642 & 2 & 1 & 2 & 8 & 2 & 5 & 2 & 2 & 2 & 2 & 0 & 0
\end{tabular}

Table 1 shows $\ell_{r}(i)$ for our example. The rounds are shown below. We discuss how the table and rounds are obtained in the following paragraph. 


\begin{tabular}{r|rrrrrrrrrrrrr}
\multicolumn{11}{c}{ rounds $r$} \\
$\ell_{r}(i)$ & 0 & 1 & 2 & 3 & 4 & 5 & 6 & 7 & 8 & 9 & 10 & 11 & 12 \\
\hline$i=1$ & $\ell$ & $\ell$ & $\ell$ & $\ell$ & $\ell-1$ & $\ell-1$ & $\ell-1$ & $\ell-4$ & $\ell-4$ & $\ell-5$ & $\ell-8$ & $\ell-8$ & $\mathbf{0}$ \\
$i=2$ & $\ell$ & 0 &. &. &. &. &. &. &. &. &. &. &. \\
$i=3$ & $\ell$ & $\ell$ & $\ell-1$ & $\ell-1$ & $\ell-2$ & 0 &. &. &. &. &. &. &. \\
$i=4$ & $\ell$ & 1 & 0 &. &. &. &. &. &. &. &. &. &. \\
$i=5$ & $\ell$ & $\ell$ & $\ell$ & $\ell$ & $\ell$ & $\ell$ & $\ell$ & $\ell$ & $\ell$ & $\ell$ & $\ell-2$ & $\mathbf{0}$ &. \\
$i=6$ & $\ell$ & 1 & 1 & 0 &. &. &. &. &. &. &. &. &. \\
$i=7$ & $\ell$ & $\ell$ & $\ell$ & $\ell$ & $\ell$ & 3 & 3 & 0 &. &. &. &. &. \\
$i=8$ & $\ell$ & 1 & 1 & 1 & 1 & 1 & 1 & 1 & 1 & $\mathbf{0}$ &. &. &. \\
$i=9$ & $\ell$ & $\ell$ & $\ell$ & $\ell$ & $\ell$ & 3 & 3 & 1 & 0 &. &. &. &. \\
$i=10$ & $\ell$ & 1 & 1 & 1 & 1 & 1 & 0 &. &. &. &. &. &. \\
$i=11$ & $\ell$ & $\ell$ & $\ell$ & $\ell$ & $\ell$ & 3 & 3 & 3 & 3 & 3 & $\mathbf{0}$ &. &. \\
$i=12$ & $\ell$ & 1 & 1 & 1 & $\mathbf{0}$ &. &. &. &. &. &. &. &.
\end{tabular}

TABLE 1 . A table of $\ell_{r}(i)$ when $n=12, k=14642$, and $\ell$ is sufficiently large.

The $\operatorname{dots}(\cdot)$ indicate the value 0 .
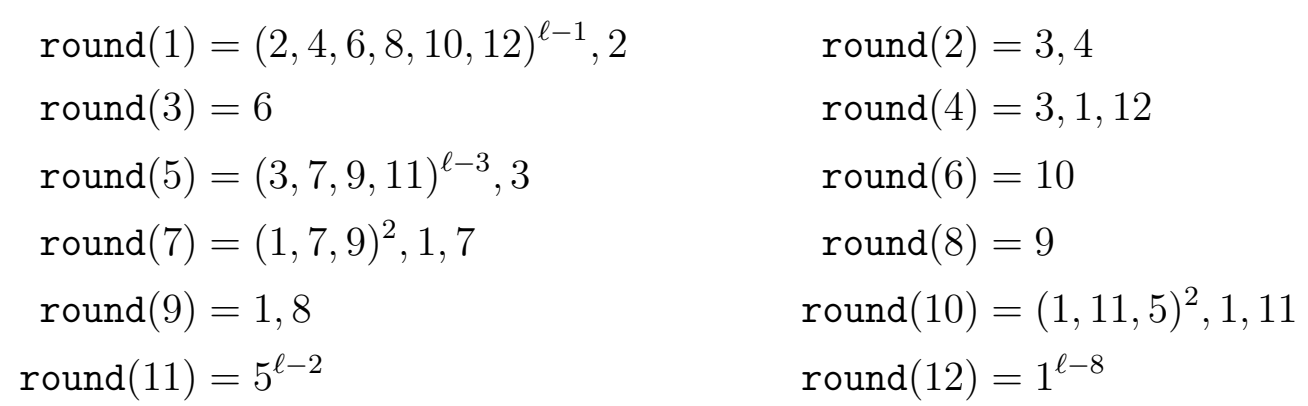

The table is constructed column-by-column. Initially, we set $\ell_{0}(i)=\ell$. To determine column $r$ from column $r-1$, one needs to know the following information:

- $\operatorname{kill}(r-1)$ (the element eliminated at the end of the previous round)

- $\ell_{r-1}(i)$ (the remaining lives at the end of the previous round)

- $k \bmod n-r+1$ (to determine the subset of elements that will be hit).

Starting at $\operatorname{kill}(r-1)$, some subset of survivors will be repeatedly and cyclicly hit until some survivor's life is reduced to one - the exact subset, call it $S$, depending on the value of $k \bmod n-r+1$. That sequence of hits can be written as $\pi^{h}$ where $\pi$ is a permutation of $S$ and $h=-1+\min \left\{\ell_{r-1}(i): i \in S\right\}$. Thereafter, the hitting continues in the order $\pi$ until the first person with one life is hit; this person is $\mathrm{kill}(r)$. This last sequence of hittings is some prefix of $\pi$, call it $\tau$. The elements of $\tau$ form some subset of $S$, call it $T$. (When $h=0$, it is natural to write $\operatorname{round}(r)=\pi^{h} \tau=\tau$. Similarly, when $\pi=\tau$, it is natural to write $\operatorname{round}(r)=\pi^{h} \tau=\pi^{h+1}$.) This technique for representing $\operatorname{round}(r)$ as a sequence of the form $\pi^{h} \tau$ can always be followed, regardless of the value of $\ell$.

It should also be clear that we can update the values of $\ell_{r}(i)$ from the information that we obtained in determining $\operatorname{round}(r)=\pi^{h} \tau$. First, if $i \notin S$, then $\ell_{r}(i)=\ell_{r-1}(i)$. If $i \in S$ and $i \in$ $\tau$, then $\ell_{r}(i)=\ell_{r}(i)-\ell_{r-1}(\operatorname{kill}(r))$. If $i \in S$ and $i \notin \tau$, then $\ell_{r}(i)=\ell_{r}(i)-\ell_{r-1}(\operatorname{kill}(r))-1$. 
Lemmas 1 and 2 show that the values of $\ell_{r}(i)$ can be uniquely expressed as $g$ or $\ell-g$ (where $g \leq F_{n+1}$ ), regardless of the specific value of $\ell \geq F_{n+2}$. That is why we left $\ell$ as a variable in Table 1. Furthermore, the values of the form $g$ are always less than the values of the form $\ell-g$ whenever $\ell \geq F_{n+2}$. Therefore, $\tau$ is uniquely determined by $\pi$. The exponent $h$ can also be uniquely expressed as $g$ or $\ell-g$, where $g \leq F_{n+1}$. In particular, the last element of $\operatorname{round}(n)$ is the final surviving element.

Theorem 1. Given fixed values of $n$ and $k$, the last surviving element $\mathrm{kill}(n)$ is constant in the feline Josephus problem for all $\ell \geq F_{n+2}$.

We now argue that the values $\ell_{r}(i)$ for $i=1,2, \ldots, n$ can be determined in time $O(n)$. First we put the non-zero values of $\ell_{r-1}(i)$ into an array of size $n-r+1$. This will allow us to advance by $k \bmod n-r+1$ in constant time, which in turn will allow us to determine the set $S$ and then the set $T$ in $O(n)$ time. It should be clear that the remaining part of the update is $O(n)$. Thus the entire table can be computed in $O\left(n^{2}\right)$ operations. We can compute $\pi$ and $\tau$ for each round at the same time, also in $O\left(n^{2}\right)$ operations.

Of course other information can be efficiently recovered from the rounds if they are stored in an appropriate data structure, say an array that indexes into the information for each round. That information would include $\pi, \tau$, and $h$. In particular, the kill sequence can be recovered in $O(n)$ time. To repeatedly determine a single value of hit $(t)$ we would want to keep a running total of the number of elements occurring in previous rounds. These totals would be stored as symbolic expressions of the form $a \ell+b$ for some integers $a$ and $b$. We could then use binary search to determine the round $r$ in which has the th hit in time $O(\log n)$. Once we have $r$, determining hit $(t)$ takes constant time.

\section{SAVing Josephus}

This section supposes that $n$ is fixed, and that Josephus is assigned to a given position around the circle. In Concrete Mathematics [10] it is shown that Josephus can always save himself in this scenario, so long as he is allowed to choose the value of $k$. We prove that this result can be extended to the feline Josephus problem by constructing a suitable value of $k$. Furthermore, the constructed value of $k$ does not depend on the specific value of $\ell$.

As in [10], our solution relies on basic principles from number theory including the Chinese Remainder Theorem (CRT), and Bertrand's Postulate. We use $a \mid b$ when $a$ divides $b$, and $\operatorname{lcm} \mathbf{S}$ for the least common multiple of a set of integers $\mathbf{S}$. After the proof, we point out why the solution in [10] does not generalize to the feline Josephus problem.

Theorem 2. Suppose $n$ and $j$ are fixed and satisfy $1 \leq j \leq n$, and $\ell$ is an arbitrary positive integer. There exists a value of $k$ such that $\operatorname{kill}(n)=j$.

Proof. If $j=n$ then $k=1$ suffices. If $j=1$ then $k=\operatorname{lcm}\{1,2, \ldots, n\}$ suffices. If $n=2$ then any even $k$ suffices for $j=1$, while any odd $k$ suffices for $j=2$. Therefore, assume $1<j<n$ and $n>2$. Bertrand's postulate implies there is a prime $p$ satisfying $n / 2<p<n$. Let $\mathbf{P}$ represent the non-empty set $\{2,3, \ldots, n\}-\{p\}$. Notice that $p \perp i$ for all $i \in \mathbf{P}$. Therefore, $p \perp \operatorname{lcm} \mathbf{P}$. We now split our construction into two cases depending on the value of $j$. The kill sequences for these two cases are illustrated in Figure 3.

Case One: $n / 2 \leq j<n$. This case will be solved by constructing a value of $k$ that results in the following hit sequence

$$
(1,2, \ldots, n)^{\ell-1}, 1,2, \ldots, n-p, j+1, j+2, \ldots, n, n-p+1, n-p+2, \ldots, j .
$$




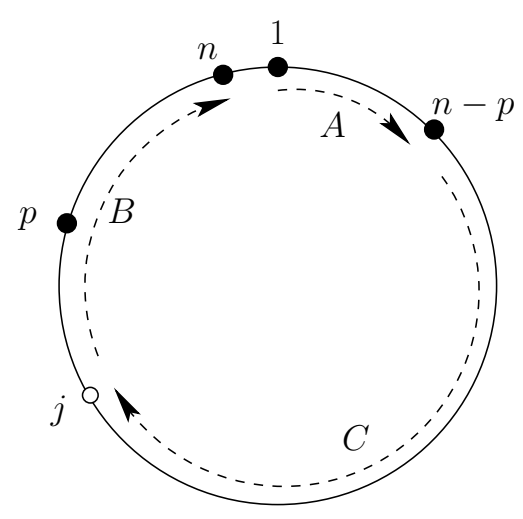

Case One.

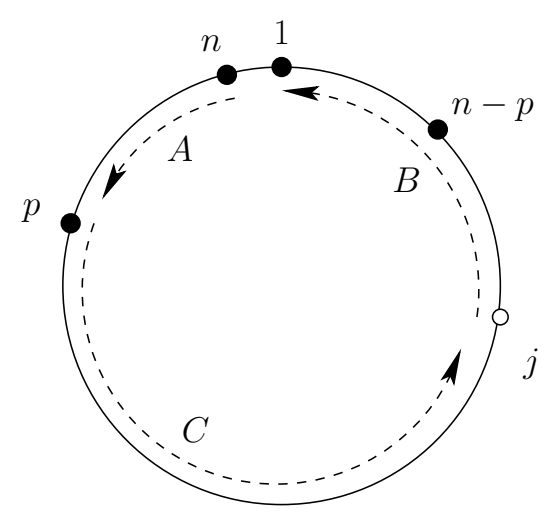

Case Two.

FiguRE 3. An illustration of the kill sequences in the two cases of Theorem

2. In each case the kill sequence is $A$, followed by $B$, followed by $C$.

This string is valid for all $\ell \geq 1$ and gives $j$ as the last surviving element. To achieve this hit sequence, we choose $k$ so that it satisfies the following congruences

$$
\begin{aligned}
& k \equiv 1 \bmod \mathrm{lcm} \mathbf{P} \\
& k \equiv j+1-n \bmod p .
\end{aligned}
$$

This choice of $k$ is possible by the CRT. The congruence (4) implies the following congruences

$$
k \equiv 1 \bmod i \text { for all } i \in \mathbf{P} \text {. }
$$

Now that the values of $k$ modulo $1,2, \ldots, n$ have been determined, we can verify that (3) is indeed the hit sequence. This will be explained in three steps. First, the prefix $(1,2, \ldots, n)^{\ell-1}, 1,2, \ldots, n-p$ follows immediately from (6). Second, (5) implies that the next element selected and removed is $j+1$. Third, (6) implies that the remaining elements selected and removed are $j+2, j+3 \ldots, n, n-p+1, n-p+2, \ldots, j$.

Case Two: $1<j<n / 2$. This case will be solved by constructing a value of $k$ that results in the following hit sequence

$$
n^{\ell},(n-1)^{\ell}, \ldots,(p+1)^{\ell}, \pi^{\ell-1}, j-1, j-2, \ldots, 1, p, p-1, \ldots, j
$$

where $\pi$ is a permutation of $\{1,2, \ldots, p\}$ beginning with $j-1$. This string is valid for all $\ell \geq 1$ and gives $j$ as the last surviving element. To achieve this hit sequence, we choose $k$ so that it satisfies the following congruences

$$
\begin{aligned}
k & \equiv 0 \bmod \operatorname{lcm} \mathbf{P} \\
k & \equiv j-1 \bmod p .
\end{aligned}
$$

This choice of $k$ is possible by the CRT. The congruence (8) implies the following congruences

$$
k \equiv 0 \bmod i \text { for all } i \in \mathbf{P} \text {. }
$$

Now that the values of $k$ modulo $1,2, \ldots, n$ have been determined, we can verify that (7) is indeed the hit sequence. This will be explained in three steps. First, the prefix $n^{\ell},(n-1)^{\ell}, \ldots,(p+1)^{\ell}$ follows immediately from (10). Second, (9) and $j-1 \perp p$ imply that the next round is $\pi^{\ell-1}, j-1$ where $\pi$ is some permutation of $\{1,2, \ldots, p\}$ that begins with $j-1$. Third, (10) implies that the remaining elements selected and removed are $j-2, j-3, \ldots, 1, p, p-1, \ldots, j$. 
This section concludes with a result that holds for the Josephus problem but not the feline Josephus problem. Consider the following hit sequences

$$
3,6,4,2,5,1 \quad 4,1,3,5,2,6 \text {. }
$$

The hit sequence on the left is for $n=6$ and $k=3$ and $\ell=1$, while the hit sequence on the right is for $n=6$ and $k=58$ and $\ell=1$. Notice that respective values in these hit sequences sum to $n+1=7$. In other words, $k=6$ and $k=58$ provide identical hit sequences except they proceed in opposite clockwise/counter-clockwise directions around the circle. More generally, $k$ and $\operatorname{lcm}\{1,2, \ldots, n\}-k+1$ provide directionally-opposite hit sequences when $n$ is fixed and $\ell=1$. On the other hand, consider the following hit sequences

$$
3,6,3,6,4,2,1,5,4,2,5,1 \quad 4,2,6,4,1,5,2,5,6,3,3,1 .
$$

The hit sequence on the left is for $n=6$ and $k=3$ and $\ell=2$, while the hit sequence on the right is for $n=6$ and $k=58$ and $\ell=2$. Notice that the hit sequences are no longer directionally-opposite, even when considering only the last surviving element. This observation led to the two-case proof of Theorem 2 that was not necessary in [10].

\section{Open Problems}

This paper has introduced a new variation of the Josephus problem known as the feline Josephus problem. Sections 2 and 3 have demonstrated that there are fun algorithmic and number theoretic problems that can be asked and solved within this generalized setting. This section suggests additional open problems arising from different perspectives, and then closes with an application of the algorithm from Section 2.1.

From an algorithmic perspective, it is natural to ask how efficiently the last surviving element $j$ can be computed for arbitrary values of $n, k$, and $\ell$. Similar questions are also natural for the kill sequence, hit sequence, and $\sigma(n)$ (discussed below).

From a mathematical perspective, it is natural to ask when the value of the last surviving element $j$ can be characterized, either directly or in terms of the original Josephus problem. For example, we have already seen that the value of $j$ does not depend on $\ell$ whenever $n$ and $k$ are relatively prime. For this reason, it may be useful to next consider situations where $n$ is a prime power that is not relatively prime with $k$.

From an artistic perspective, it is interesting to consider the spiral drawings found in Figures 1 and 2. Determining the number of spirals that appear in one of these drawings is equivalent to asking the following question: Given $n, k$, and $\ell$, how many times does one have to travel around the circle until the last surviving element is obtained.

From the perspective of a person who wishes to avoid any pain as long as possible, what can be proven about the last person to be hit for the first time? In the original $\ell=1$ Josephus problem, the last person to be hit is $\operatorname{kill}(n)$. However, this is not necessarily true for $\ell>1$. For example, the end of hit sequence for $n=12, k=14642$, and $\ell>8$ is restated from $(2)$ is $\ldots 10,(1,7,9)^{2}, 1,7,9,1,8,(1,11,5)^{2}, 1,11,5^{\ell-2}, 1^{\ell-8}$. Notice that $\operatorname{kill}(n)=1$, whereas element 5 is the last element to be hit for the first time (in round 10). To provoke further questions, consider the following hit sequence for $n=6, k=10$, and $\ell \geq 2$ :

$$
(426)^{\ell-1} 4,3^{\ell}, 6,12,1^{\ell-1}, 5^{\ell} .
$$

Notice that the last surviving element 5 is not hit until the final round. When do situations like this occur, and is there at least one such value of $k$ for every value of $n$ ? 
Perhaps the most natural open problem is motivated by running the algorithm outlined in Section 2.1. For example, Table 2 contains output for $n=3,4,5,6$ and all $k$ modulo $\operatorname{lcm}\{1,2, \ldots, n\}$. From this table it is clear that the Fibonacci bound discussed in Section 2 is not tight. For example, in the $n=6$ column of Table $2, \ell-2$ is the exponent with the lowest negative integer. Therefore, all of the hit sequences for $n=6$ are valid for $\ell \geq 3$, as opposed to the bound $\ell \geq F_{8}=55$ from Theorem 1. More generally, let $\sigma(n)$ be the lowest negative integer exponent in a hit sequence for $n$ elements generated by our algorithm. The following values show $\sigma(n)$ in comparison with $F_{n+2}$ for $n=1,2, \ldots, 14$.

\begin{tabular}{|c|rrrrrrrrrrrrrr|}
\hline$n=$ & 1 & 2 & 3 & 4 & 5 & 6 & 7 & 8 & 9 & 10 & 11 & 12 & 13 & 14 \\
\hline$\sigma(n)$ & 1 & 1 & 1 & 1 & 1 & 3 & 3 & 4 & 6 & 6 & 6 & 9 & 9 & 11 \\
$F_{n+2}$ & 2 & 3 & 5 & 8 & 13 & 21 & 34 & 55 & 89 & 144 & 233 & 377 & 610 & 987 \\
\hline
\end{tabular}

Notice that for small values of $n$, the correct bound for $\sigma(n)$ appears to be closer to $n$ than $F_{n+2}$. Tightening the bound on $\sigma(n)$ promises further number theoretic and algorithmic fun.

\section{REFERENCES}

[1] W. W. Rouse Ball and H. S. M. Coxeter. Mathematical Recreations and Essays. Dover Publications, 1987.

[2] N. L. Biggs. The roots of combinatorics. Historia Math., 6(2):109-136, 1979.

[3] Sarah Breede and Christy Finch. Fixed points of the $(n, 3)$-Josephus permutations. The Journal of the Summer Undergraduate Mathematical Science Research Institute (SUMSRI), 2001.

[4] Sarah Burke and Robert Davis. The ABBA modification of the Josephus problem. The Journal of the Summer Undergraduate Mathematical Science Research Institute (SUMSRI), 2001.

[5] Girolamo Cardano. Practica Arithmetice et Mensurandi Singularis. 1539.

[6] Ledah Casburn and Tuyet-Linh Phan. The orthogonal Josephus problem. The Journal of the Summer Undergraduate Mathematical Science Research Institute (SUMSRI), 2001.

[7] James Dowdy and Michael E. Mays. Josephus permutations. J. Combin. Math. Combin. Comput., 6:125-130, 1989.

[8] Martin Gardner. Mathematical Puzzles of Sam Loyd, volume 2. Dover Publications, 1960.

[9] Fatih Gelgi and Errol L. Lloyd. Josephus: an improved algorithm for finding the sole survivor, unpublished manuscript. 2002.

[10] R. L. Graham, D. E. Knuth, and O. Patashnik. Concrete Mathematics. Adison Wesley, 1994.

[11] Lorenz Halbeisen and Norbert Hungerbhler. The Josephus problem. J. Théor. Nuombres Bordeaux., 9:303-318, 1997.

[12] I.N. Herstein and I. Kaplansky. Matters Mathematical. Harper \& Row, 1974.

[13] F. Jakobczyk. On the generalized Josephus problem. Glasgow Mathematical Journal, 14:168-173, 1973.

[14] Titus Flavius Josephus. The Jewish War. 75. ISBN 0-14-044420-3.

[15] Errol L. Lloyd. An $o(n \log m)$ algorithm for the Josephus problem. J. Algorithms, 4(3):262-270, 1983.

[16] Andrew M. Odlyzko and Herbert S. Wilf. Functional iteration and the Josephus problem. Glasgow Mathematical Journal, 33(2):235-240, 1991.

[17] W. J. Robinson. The Josephus problem. Math. Gaz., 4:47-52, 1960.

[18] Susan H. Rodger. Using hands-on visualizations to teach computer science from beginning courses to advanced courses, 2002.

[19] N. J. A. Sloane. The on-line encyclopedia of integer sequences. http://www.research.att.com/ njas/ sequences/A003418.

[20] N. Theérialut. Generalizations of the Josephus problem. Util. Math., 58:161-173, 2000.

[21] D. Woodhouse. The extended Josephus problem. Rev. Mat. Hisp.-Amer., 4(33):207-218, 1973.

Dept. of Computer Science, University of Victoria, Canada.

Dept. of Computer Science, University of Victoria, Canada. 


\begin{tabular}{|c|c|c|c|c|}
\hline & $n=3$ & $n=4$ & $n=5$ & $n=6$ \\
\hline$k=1$ & $(123)^{\ell-1} 1,2,3$ & $(1234)^{\ell-1} 1,2,3,4$ & $(12345)^{\ell-1} 1,2,3,4,5$ & $(123456)^{\ell-1} 1,2,3,4,5,6$ \\
\hline$k=2$ & $(213)^{\ell-1} 2,1,3$ & $(24)^{\ell-1} 2,4,3^{\ell}, 1^{\ell}$ & $(24135)^{\ell-1} 2,4,1,5,3$ & $(246)^{\ell-1} 2,4,6,(315)^{\ell-1} 3,1,5$ \\
\hline$k=3$ & $3^{\ell},(12)^{\ell-1} 1,2$ & $(3214)^{\ell-1} 3,2,4,1$ & $(31425)^{\ell-1} 3,1,5,2,4$ & $(36)^{\ell-1} 3,6,(4215)^{\ell-1} 4,2,5,1$ \\
\hline$k=4$ & $(123)^{\ell-1} 1,3,2$ & $4^{\ell},(123)^{\ell-1} 1,3,2$ & $(43215)^{\ell-1} 4,3,5,2,1$ & $(426)^{\ell-1} 4,2,1^{\ell}, 356,5^{\ell-1}, 3^{\ell-1}$ \\
\hline$k=5$ & $(213)^{\ell-1} 2,3,1$ & $(1234)^{\ell-1} 1,3,4,2$ & $5^{\ell},(1234)^{\ell-1} 1,3,4,2$ & $(543216)^{\ell-1} 5,4,6,2,3,1$ \\
\hline$k=6$ & $3^{\ell}, 2^{\ell}, 1^{\ell}$ & $(24)^{\ell-1} 2,1^{\ell}, 4,3^{\ell}$ & $(12345)^{\ell-1} 1,3,2,5,4$ & $6^{\ell},(12345)^{\ell-1} 1,3,2,5,4$ \\
\hline$k=7$ & & $(3214)^{\ell-1} 3,4,1,2$ & $(24135)^{\ell-1} 2,5,1,3,4$ & $(123456)^{\ell-1} 1,3,6,2,4,5$ \\
\hline$k=8$ & & $4^{\ell},(213)^{\ell-1} 2,1,3$ & $(31425)^{\ell-1} 3,2,5,4,1$ & $(246)^{\ell-1} 2,536,5^{\ell-1}, 314,3^{\ell-2}, 1^{\ell-1}$ \\
\hline$k=9$ & & $(1234)^{\ell-1} 1,4,2,3$ & $(43215)^{\ell-1} 4,5,3,1,2$ & $(36)^{\ell-1} 3,16,(1245)^{\ell-2} 1,5^{2},(24)^{1} 2,4$ \\
\hline$k=10$ & & $(24)^{\ell-1} 2,34,3^{\ell-1}, 1^{\ell}$ & $5^{\ell},(24)^{\ell-1} 2,34,3^{\ell-1}, 1^{\ell}$ & $(426)^{\ell-1} 4,3^{\ell}, 6,12,1^{\ell-1}, 5^{\ell}$ \\
\hline$k=11$ & & $(3214)^{\ell-1} 3,1,2,4$ & $(12345)^{\ell-1} 1,4,2,3,5$ & $(543216)^{\ell-1} 5,6,3,1,2,4$ \\
\hline$k=12$ & & $4^{\ell}, 3^{\ell}, 2^{\ell}, 1^{\ell}$ & $(24135)^{\ell-1} 2,1,5,4,3$ & $6^{\ell},(24135)^{\ell-1} 2,1,5,4,3$ \\
\hline$k=13$ & & & $(31425)^{\ell-1} 3,4,5,1,2$ & $(123456)^{\ell-1} 1,4,5,6,2,3$ \\
\hline$k=14$ & & & $(43215)^{\ell-1} 4,1,3,2,5$ & $(246)^{\ell-1} 2,6,(35)^{\ell-1} 3,5,4,1^{\ell}$ \\
\hline$k=15$ & & & $5^{\ell},(3214)^{\ell-1} 3,2,4,1$ & $(36)^{\ell-1} 3,2^{\ell}, 6,5^{\ell},(14)^{\ell-1} 1,4$ \\
\hline$k=16$ & & & $(12345)^{\ell-1} 1,5,2,4,3$ & $(426)^{\ell-1} 4,56,5^{\ell-1}, 12,1^{\ell-1}, 3^{\ell}$ \\
\hline$k=17$ & & & $(24135)^{\ell-1} 2,3,5,1,4$ & $(543216)^{\ell-1} 5,1,2,4,6,3$ \\
\hline$k=18$ & & & $(31425)^{\ell-1} 3,5,4,2,1$ & $6^{\ell},(31425)^{\ell-1} 3,5,4,2,1$ \\
\hline$k=19$ & & & $(43215)^{\ell-1} 4,2,3,5,1$ & $(123456)^{\ell-1} 1,5,3,4,6,2$ \\
\hline$k=20$ & & & $5^{\ell}, 4^{\ell},(213)^{\ell-1} 2,1,3$ & $(246)^{\ell-1} 2,1^{\ell}, 6,4,3^{\ell}, 5^{\ell}$ \\
\hline$k=21$ & & & $(12345)^{\ell-1} 1,2,5,3,4$ & $(36)^{\ell-1} 3,456,(1245)^{\ell-2} 124,2,5,1$ \\
\hline$k=22$ & & & $(24135)^{\ell-1} 2,4,5,3,1$ & $(426)^{\ell-1} 4,6,2,(351)^{\ell-1} 3,1,5$ \\
\hline$k=23$ & & & $(31425)^{\ell-1} 3,1,4,5,2$ & $(543216)^{\ell-1} 5,2,6,3,4,1$ \\
\hline$k=24$ & & & $(43215)^{\ell-1} 4,3,2,1,5$ & $6^{\ell},(43215)^{\ell-1} 4,3,2,1,5$ \\
\hline$k=25$ & & & $5^{\ell},(1234)^{\ell-1} 1,2,3,4$ & $(123456)^{\ell-1} 1,6,2,3,4,5$ \\
\hline$k=26$ & & & $(12345)^{\ell-1} 1,3,5,4,2$ & $(246)^{\ell-1} 2,34,6,(315)^{\ell-2} 3,1^{2}, 5^{2}$ \\
\hline$k=27$ & & & $(24135)^{\ell-1} 2,5,4,1,3$ & $(36)^{\ell-1} 3,5146,(4215)^{\ell-2} 4,2^{2}, 5,1$ \\
\hline$k=28$ & & & $(31425)^{\ell-1} 3,2,4,1,5$ & $(426)^{\ell-1} 4,152,1^{\ell-1}, 356,5^{\ell-2}, 3^{\ell-1}$ \\
\hline$k=29$ & & & $(43215)^{\ell-1} 4,5,2,3,1$ & $(543216)^{\ell-1} 5,3,4,1,2,6$ \\
\hline$k=30$ & & & $5^{\ell},(24)^{\ell-1} 2,1^{\ell}, 4,3^{\ell}$ & $6^{\ell}, 5^{\ell},(24)^{\ell-1} 2,1^{\ell}, 4,3^{\ell}$ \\
\hline$k=31$ & & & $(12345)^{\ell-1} 1,4,5,2,3$ & $(123456)^{\ell-1} 1,2,5,6,3,4$ \\
\hline$k=32$ & & & $(24135)^{\ell-1} 2,1,4,3,5$ & $(246)^{\ell-1} 2,4,3^{\ell}, 6,5^{\ell}, 1^{\ell}$ \\
\hline$k=33$ & & & $(31425)^{\ell-1} 3,4,2,5,1$ & $(36)^{\ell-1} 3,6,(1245)^{\ell-1} 1,5,2,4$ \\
\hline$k=34$ & & & $(43215)^{\ell-1} 4,1,2,5,3$ & $(426)^{\ell-1} 4,2,(51)^{\ell-1} 5,6,3^{\ell}, 1$ \\
\hline$k=35$ & & & $5^{\ell},(3214)^{\ell-1} 3,1,2,4$ & $(543216)^{\ell-1} 5,4,2,6,1,3$ \\
\hline$k=36$ & & & $(12345)^{\ell-1} 1,5,4,3,2$ & $6^{\ell},(12345)^{\ell-1} 1,5,4,3,2$ \\
\hline$k=37$ & & & $(24135)^{\ell-1} 2,3,4,5,1$ & $(123456)^{\ell-1} 1,3,4,5,6,2$ \\
\hline$k=38$ & & & $(31425)^{\ell-1} 3,5,2,1,4$ & $(246)^{\ell-1} 2,536,(35)^{\ell-2} 3,5,4,1^{\ell}$ \\
\hline$k=39$ & & & $(43215)^{\ell-1} 4,2,1,3,5$ & $(36)^{\ell-1} 3,16,(4215)^{\ell-2} 421,5^{2}, 2,4$ \\
\hline$k=40$ & & & $5^{\ell}, 4^{\ell},(123)^{\ell-1} 1,3,2$ & $(426)^{\ell-1} 4,3^{\ell}, 2,56,5^{\ell-1}, 1^{\ell}$ \\
\hline$k=41$ & & & $(12345)^{\ell-1} 1,2,4,5,3$ & $(543216)^{\ell-1} 5,6,1,3,4,2$ \\
\hline$k=42$ & & & $(24135)^{\ell-1} 2,4,3,1,5$ & $6^{\ell},(24135)^{\ell-1} 2,4,3,1,5$ \\
\hline$k=43$ & & & $(31425)^{\ell-1} 3,1,2,4,5$ & $(123456)^{\ell-1} 1,4,2,3,5,6$ \\
\hline$k=44$ & & & $(43215)^{\ell-1} 4,3,1,5,2$ & $(246)^{\ell-1} 2,6,5^{\ell}, 314,3^{\ell-1}, 1^{\ell-1}$ \\
\hline$k=45$ & & & $5^{\ell},(1234)^{\ell-1} 1,4,2,3$ & $(36)^{\ell-1} 3,2^{\ell}, 456,5^{\ell-1},(14)^{\ell-1}, 1$ \\
\hline$k=46$ & & & $(12345)^{\ell-1} 1,3,4,2,5$ & $(426)^{\ell-1} 4,56,2,(351)^{\ell-2} 35,3,1^{2}$ \\
\hline$k=47$ & & & $(24135)^{\ell-1} 2,5,3,4,1$ & $(543216)^{\ell-1} 5,1,4,2,3,6$ \\
\hline$k=48$ & & & $(31425)^{\ell-1} 3,2,1,5,4$ & $6^{\ell},(31425)^{\ell-1} 3,2,1,5,4$ \\
\hline$k=49$ & & & $(43215)^{\ell-1} 4,5,1,2,3$ & $(123456)^{\ell-1} 1,5,6,2,3,4$ \\
\hline$k=50$ & & & $5^{\ell},(24)^{\ell-1} 2,4,3^{\ell}, 1^{\ell}$ & $(246)^{\ell-1} 2,1^{\ell}, 4,6,5^{\ell}, 3^{\ell}$ \\
\hline$k=51$ & & & $(12345)^{\ell-1} 1,4,3,5,2$ & $(36)^{\ell-1} 3,456,(4215)^{\ell-2} 4,2^{2}, 5,1^{2}$ \\
\hline$k=52$ & & & $(24135)^{\ell-1} 2,1,3,5,4$ & $(426)^{\ell-1} 4,6,5^{\ell}, 12,1^{\ell-1}, 3^{\ell}$ \\
\hline$k=53$ & & & $(31425)^{\ell-1} 3,4,1,2,5$ & $(543216)^{\ell-1} 5,2,3,6,1,4$ \\
\hline$k=54$ & & & $(43215)^{\ell-1} 4,1,5,3,2$ & $6^{\ell},(43215)^{\ell-1} 4,1,5,3,2$ \\
\hline$k=55$ & & & $5^{\ell},(3214)^{\ell-1} 3,4,1,2$ & $(123456)^{\ell-1} 1,6,4,5,2,3$ \\
\hline$k=56$ & & & $(12345)^{\ell-1} 1,5,3,2,4$ & $(246)^{\ell-1} 2,34,3^{\ell-1}, 6,5^{\ell}, 1^{\ell}$ \\
\hline$k=57$ & & & $(24135)^{\ell-1} 2,3,1,4,5$ & $(36)^{\ell-1} 3,5146,(1245)^{\ell-2} 1,5,24,2$ \\
\hline$k=58$ & & & $(31425)^{\ell-1} 3,5,1,4,2$ & $(426)^{\ell-1} 4,152,(51)^{\ell-2} 5,6,3^{\ell}, 1$ \\
\hline$k=59$ & & & $(43215)^{\ell-1} 4,2,5,1,3$ & $(543216)^{\ell-1} 5,3,1,4,6,2$ \\
\hline$k=60$ & & & $5^{\ell}, 4^{\ell}, 3^{\ell}, 2^{\ell}, 1^{\ell}$ & $6^{\ell}, 5^{\ell}, 4^{\ell}, 3^{\ell}, 2^{\ell}, 1^{\ell}$ \\
\hline
\end{tabular}

TABLE 2. Hit sequences for $3 \leq n \leq 6,1 \leq k \leq \operatorname{lcm}\{1,2, \ldots, n\}$ and $\ell \geq$ $F_{n+2}$. Commas appear only at the end of each round. 